\title{
Personal Exposure to Ultrafine Particles, Black Carbon and PM 2.5 in Different Microenvironments
}

\author{
Josef Cyrys ${ }^{1}$, J. Gu ${ }^{1}$, M. Pitz ${ }^{2}$, and A. Peters ${ }^{1}$ \\ 1 Helmholtz-Zentrum für Gesundheit und Umwelt (HMGU) München, Institute for Epidemiology, München, Germany, \\ E-mail: cyrys@helmholtz-muenchen.de \\ 2 Bavarian Agency for Environment, Augsburg, Germany
}

Exposure assessment studies have shown that air pollution measured by fixed monitors at residential locations may not adequately represent individual exposures, especially for times spent away from home. In this study we characterized personal exposures in specific micro-environments to particulate air pollutants from different sources. During three periods, exposure data were collected by a single researcher equipped with personal monitors for continuous measurements of particle number concentration (PNC), black smoke (BS) and PM 2.5. As pedestrian or passenger in public transportation he was taking the same route including major roads, urban background and industrial areas every day. His position was recorded by a GPS device.

We have shown that personal exposure to PM 2.5, BC and PNC is always higher than ambient concentration measured at a fixed monitoring station. BC and PNC levels were considerable higher in traffic related scenarios compared to scenarios in residential areas. In contrast, PM 2.5 was found less variable in different scenarios. Moreover, daily averaged personal and ambient concentrations are strongly correlated (PM $2.5 \approx B C>P N C$ ). This suggests that the day-to-day variation of personal exposure to air pollutants may be sufficiently reflected by a fixed measurement station for use in epidemiological short-term studies. The obtained results provide insight into the potential air pollution levels to which people could be exposed and suggest that personal exposure might be considered in future epidemiological studies in addition to data from the fixed monitoring station in order to get more reliable estimates concerning people's total exposure to UFP, BS and PM 2.5 , especially when being in traffic or in a near-road environment. 